\title{
Application of $\mathrm{Nb}_{3} \mathrm{Sn}$ Superconductors in High-Field Accelerator Magnets
}

\author{
Andries den Ouden, Sander Wessel, Erik Krooshoop and Herman ten Kate. \\ Low Temperature Division, University of Twente, P.O. Box 217, 7500 AE Enschede, The Netherlands
}

\begin{abstract}
Last year a record central field of $11 \mathrm{~T}$ at first excitation at $4.4 \mathrm{~K}$ has been achieved with the experimental LHC model dipole magnet MSUT by utilising a high $J_{c}$ powderin-tube $\mathrm{Nb}_{3} \mathrm{Sn}$ conductor. This is the first real breakthrough towards fields well above $10 \mathrm{~T}$ at $4 \mathrm{~K}$. The clear influence of magnetisation and coupling currents on the field quality, the quench behaviour and the temperature development in the coils has been measured and is discussed. For application in highfield accelerator magnets (10-15 $\mathrm{T}$ dipoles, 300-400 T/m quadrupoles) these experimental results clearly reveal the potential, the present limitations and the necessary improvements of $\mathrm{Nb}_{3} \mathrm{Sn}$ technology with respect to strand, cable and coil design and manufacturing. A brief review of developments in this field is presented. The focus is on accelerator dipole magnets but the key issues for quadrupole magnets are quite similar
\end{abstract}

\section{INTRODUCTION}

The development of accelerator magnets (dipoles and quadrupoles) is focused on high-field strength c.q. high current density, field quality (normalised higher order multipoles $<10^{-4}$ ), reliable operation (mechanical and thermal stability) and high quality large scale production. The present practical field limit for $\mathrm{NbTi}$ magnets operating at $2 \mathrm{~K}$ amounts to about $8.5 \mathrm{~T}$. The relation between design and manufacturing parameters and the quench behaviour (location, ageing and thermal cycling effects, thermal or mechanical limitations) is not well understood yet [1], which impedes large scale production. The amplitude and the time dependent behaviour of the undesired higher order multipoles as well as the ramp rate sensitivity can be described quite well by modelling the interstrand coupling currents and the boundary induced coupling currents [2]. The necessary control of these currents and the consequences for cable stability is still under investigation [3].

To attain a field of 10-15 $\mathrm{T}$ presently only $\mathrm{Nb}_{3} \mathrm{Sn}$ conductors, operating at $4.4 \mathrm{~K}$, are available in satisfactory quality and quantity. Apart from the general issues concerning highfield accelerator magnets mentioned above, typical $\mathrm{Nb}_{3} \mathrm{Sn}$ related difficulties have to be taken into account. All coil manufacturing steps concerning winding, heat treatment at $675^{\circ} \mathrm{C}$, the internal splice, protection heaters, resin impregnation, mechanical support and assembly are strongly related to each other and require a more integrated and careful approach than in the case of NbTi coils.

Manuscript received august 26, 1996

This work has been supported, in part, by the Netherlands Technology Foundation STW.

The $\mathrm{Nb}_{3} \mathrm{Sn}$ dipole magnet program is a collaboration between the University of Twente, STW, NIKHEF Amsterdam and CERN Geneva.
On strand and cable level the key issues comprise the reduction of $I_{c}$, due to cabling (filament damage), transverse stress sensitivity of $J_{c}$, the still existing controversy of a high $J_{c}$ versus filament size, heat resistant and thermally conductive electrical insulation, control of the interstrand crossing and adjacent resistances $R_{c}$ and $R_{u}$ in relation to the thermal and electrical stability and the low normal zone propagation.

To illustrate the present status and the potential of $\mathrm{Nb}_{3} \mathrm{Sn}$ accelerator magnets, the experimental results of the successful program to realise a 1 meter $11 \mathrm{~T}$ single aperture $\mathrm{Nb}_{3} \mathrm{Sn}$ dipole magnet MSUT are discussed. In this program the emphasis has been put on the main challenge, namely to increase the field strength by exploiting the high $J_{c^{\prime}}$ of the powder-in-tube $\mathrm{Nb}_{3} \mathrm{Sn}$ conductor [4] and developing $\mathrm{Nb}_{3} \mathrm{Sn}$ dedicated design and manufacturing concepts.

\section{MSUT SYSTEM CHARACTERISTICS}

Table 1 summarises the most important coil parameters. Note that the inner layer cable is the largest Rutherford cable ever made for accelerator magnets.

In fig. 1 the single pole conductor lay-out in the splice region is depicted schematically. Instead of a ramp splice the inner and outer layer are connected in the pole plane by prereacted $\mathrm{Nb}_{3} \mathrm{Sn}$ wires which are soldered with $\mathrm{SnAg}$ to a $2 \mathrm{~mm}$ thick copper plate. This splice configuration eases layer handling and ensures continuity of the mechanical support in the pole plane. As a consequence of this configuration the outer layer conductors in this region experience a $0.15 \mathrm{~T}$ higher maximum field than in the normal cross section. Fig. 2 shows the non-copper $J_{c}$ of the conductors before and after cabling as well as the loadlines for both layers to a maximum central field of $11 \mathrm{~T}$. The significant degradation due to cabling of the outer layer conductors, which reduces the $J_{c^{-}}$

TABLE I

MSUT COIL PARAMETERS

\begin{tabular}{lccc}
\hline & Inner cable & Outer cable & \\
\hline & & & \\
Dimensions (bare) & $1.98 / 2.47 \times 21.7$ & $1.54 / 1.82 \times 17.4$ & $\mathrm{~mm}$ \\
Number of strands & 33 & 33 & \\
Strand diameter & 1.26 & 1.00 & $\mathrm{~mm}$ \\
Twist pitch & 147 & 146 & $\mathrm{~mm}$ \\
Number of filaments & 192 & 192 & \\
Filament diameter & 42 & 32 & $\mu \mathrm{m}$ \\
Filament twist pitch & 30 & 30 & $\mathrm{~mm}$ \\
Cu percentage & 56 & 56 & $\%$ \\
Insulation thickness & 0.14 & 0.14 & $\mathrm{~mm}$ \\
Max field in cable & 11.3 & 9.4 & $\mathrm{~T}$ \\
Max. eq. stress & 140 & 120 & $\mathrm{MPa}$ \\
\hline
\end{tabular}




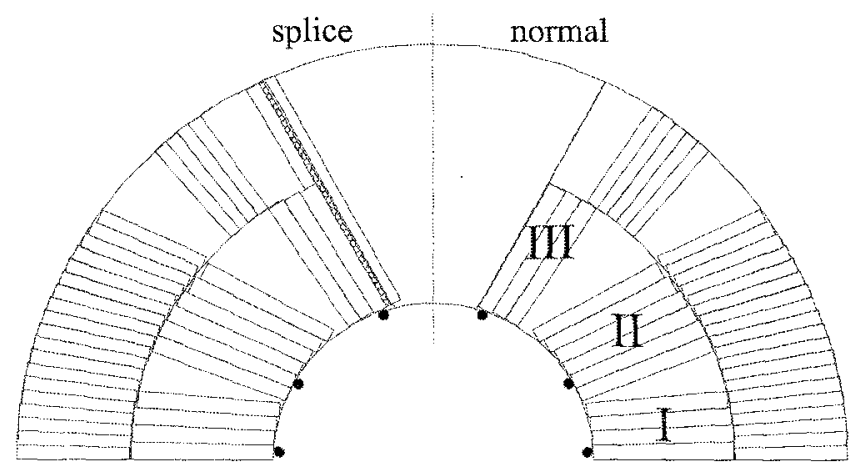

Fig. 1. Conductor lay-out in the splice region of a single pole, which extends to $30 \mathrm{~cm}$ of the straight part. Also shown are the 6 thermometer positions and the inner layer block labels. The bore dimeter is $50 \mathrm{~mm}$.

margin in the splice region from about $35 \%$ to $10 \%$, was not expected from earlier experiences with PIT-conductor Rutherford cables [5]. The cabling degradation of the inner layer conductor is less severe, though the initial strand $J_{c}$ is quite low due to a non-optimised strand lay-out. To exploit the high $J_{c}$-potential of $\mathrm{Nb}_{3} \mathrm{Sn}$ conductors for a particular application a dedicated optimisation process with respect to strand and cable lay-out seems to be imperative. This is an important observation for the development of optimally performing $\mathrm{Nb}_{3} \mathrm{Sn}$ cables. In the MSUT program the optimisation has not been carried out due to time constraints. More details of the electromagnetic and mechanical design of the MSUT magnet are described elsewhere [6], [7], [8].

\section{A. Manufacturing experiences}

As important as the design and the conductor performance is the manufacturing of sound $\mathrm{Nb}_{3} \mathrm{Sn}$ coils and their me-

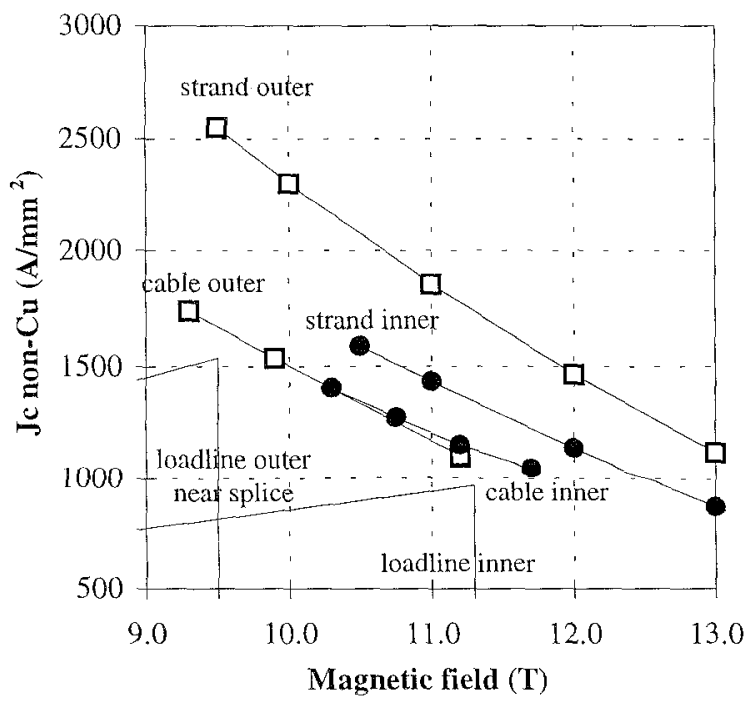

Fig. 2. Measured non-copper critical current density versus magnetic field before (strand) and after cabling (cable) for both layer conductors. Also shown are the loadlines to a maximum central field of $11 \mathrm{~T}$. chanical support. The only insulation material that appeared to be reliable during winding of the large inner cables was a folded mica/glass sheet wrapped with a glass fibre tape [9]. Even at the beginning and the end of the turns where severe cable deformation occurred during winding and pressing, this insulation system did not suffer from any turn-to-turn shorts.

For controlling the shape and position of such large cables the empirically developed method for coil head support elements is to our opinion still the only way to realise reliable turns [8]. Though perhaps more time consuming, it assures continuous support between the straight section and the coil head, good support in the turns itself and flexibility of the coil head during the heat treatment. Especially this last issue is important because the heat treatment gives rise to complete stress release and a significant axial expansion of the coils. Irrespective what support elements are used, this expansion manifest itself in the heads as large gaps between turns and support elements. This approach especially facilitates gap filling with glass fibres afterwards to ensure complete support.

Shrinking of the warm $\left(225^{\circ} \mathrm{C}\right)$ ring shaped collars around the finished coils was successful though heating of the coils to about $90^{\circ} \mathrm{C}$, at which temperature yielding of the epoxy starts, could not be prevented. This resulted in a reduced prestress after collaring, which could be compensated for by adapting both the collar-yoke and the yoke-outer shell interference. This collaring technique will be more difficult but is still worth developing for longer coils.

\section{EXPERIMENTAL RESULTS}

The magnet has been tested for 3 weeks at the model magnet test facility at CERN at a temperature of $4.4 \mathrm{~K}$. For protective and diagnostical purposes the magnet is equipped with meander shaped stainless steel foil quench-protection heaters at the inner and outer layers, many voltage taps at the inner and outer layers, spot heaters and 4 point germanium resistance thermometers at the inner layer bore (see fig. 1). Magnetic field measurements have been performed using rotating pick-up coils [10]. A static pick-up coil set is used for quench localisation [11]. The integrated hysteretic and coupling losses during field cycles are measured electrically [12].

To protect the coils from overheating during this first test run nearly all stored energy is extracted after each quench and dumped into an external resistor.

\section{A. Quench characteristics}

At the very first high-field ramp the current was ramped with $2 \mathrm{~A} / \mathrm{s}$ from $15 \mathrm{kA}$ to the first quench at a current of $18.7 \mathrm{kA}$, corresponding to a record central dipole field of 11.03 T. At intermediate field levels at least up to $10.7 \mathrm{~T}$ the ramp could be halted to perform field measurements. Immediately hereafter a second run resulted in a quench at $18.5 \mathrm{kA}$ $(10.92 \mathrm{~T})$, about $1.5 \%$ lower than the first quench. After 3 weeks of experimenting a final high current ramp resulted in a quench at $18.4 \mathrm{kA}(10.86 \mathrm{~T})$. Fig. 3 shows the measured relation between central field and current. 


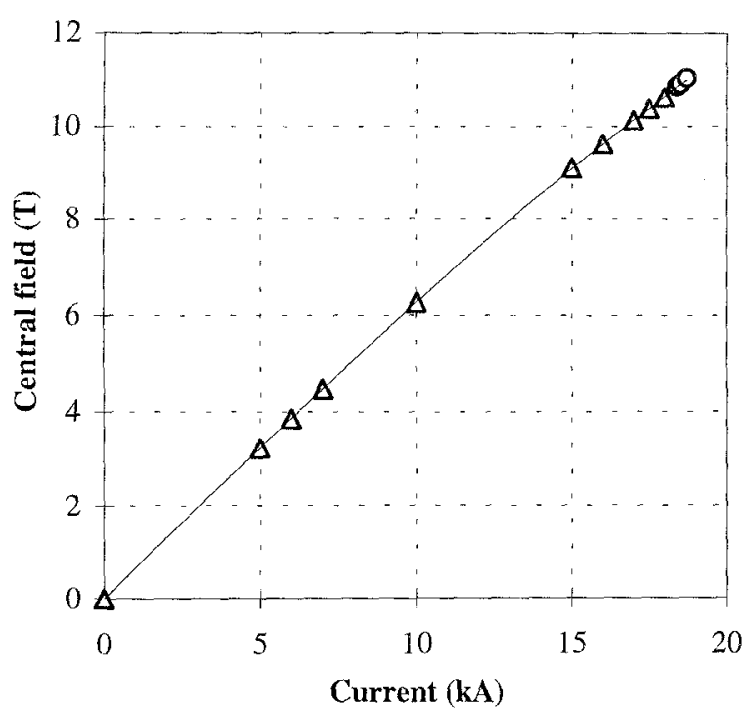

Fig. 3. Measured central field of the $\mathrm{Nb}_{3} \mathrm{Sn}$ dipole magnet MSUT as a function of the operating current at $4.4 \mathrm{~K}$. The line is a quadratic fit to the measured points. Open circles indicate the quenches.

All quenches occurred in the outer layer of the same pole in the splice region, where the field-current conditions are most unfavourable (see fig. 2). At maximum field the transverse stress on the wide side of the inner layer amounts to 120 $140 \mathrm{MPa}$, depending on the frictional effects in the mechanical support system. This indicates, that at this stress level the non-permanent $J_{c}$ reduction of the inner cable is less than $10 \%\left(J_{c}\right.$ margin in fig. 2$)$, a typical behaviour of a good performing $\mathrm{Nb}_{3} \mathrm{Sn}$ Rutherford cable [13]. The slight decrease of the successive quench currents might point to coil damage due to the sudden stress release during the quenches. This issue will be investigated during a second test run.

It is important to note that this quench behaviour without any training effects is characteristic for good performing fully potted magnets. This has also been observed in the first $\mathrm{Nb}_{3} \mathrm{Sn}$ LHC dipole magnet, which reached $9.5 \mathrm{~T}$ in only a few quenches [14]. This is a remarkable difference with dry $\mathrm{NbTi}$ dipole magnets operating at $2 \mathrm{~K}$, that need many training quenches to reach a plateau close to the cable $I_{c}$.

\section{$B$. Losses, field quality and ramp rate sensitivity}

Though the main target in the MSUT program was to reach a field strength of $11 \mathrm{~T}$, the field quality is an important operating parameter for accelerator magnets. Presently the demands are, that the higher order normalised multipoles $\mathrm{B}_{\mathrm{n}} / \mathrm{B}_{1}$ (European convention: $\mathrm{B}_{1}=$ normal dipole) are smaller than $10^{-4}$ at a bore radius of $10 \mathrm{~mm}$.

At accelerator injection field of about $0.4 \mathrm{~T}$ the most significant field errors originate from magnetisation currents, manufacturing tolerances and a non-uniform current distribution due to the soldering connections.

At maximum operating field the magnetisation contribution is negligible and eventually additional distortion due to iron saturation arises.

During ramping of the field, interstrand coupling currents (ISCCs) and boundary induced coupling currents (BICCs) cause additional field errors. The influence of interfilament coupling currents can be neglected [2].

Generally the ISCCs are proportional to $d B_{\perp} / d t, w, L_{p}, N$ and $R_{c}^{-1}$, with $B_{\perp}$ the field component perpendicular to the wide side of the cable, $w$ the cable width, $L_{p}$ the cable twist length, $N$ the number of strands and $R_{c}$ the resistance between crossing strands. The typical ISCC distribution gives rise to normal odd multipole components. Their characteristic time constant $\tau_{\text {is }}$ varies from 1-50 seconds. The associated losses are proportional to $\left(d B_{\perp} / d t\right)^{2}, w^{2}, N^{2}, R_{c}^{-1}$ and $L_{p}$.

The BICCs are induced by (steep) gradients or nonuniformities of $\left(d B_{\perp} / d t\right)$ and $R_{c}$ along the cable. This is definitely the case in the coil heads and the internal splice of dipole magnets. The BICC value is proportional to $w, R_{c}{ }^{-1}$ and a characteristic length $L_{b i}$. In contrast to ISCC's they run mainly in the filaments over lengths varying from a few times $L_{p}$ to the whole cable length and are characterised by time constant that varies from $10^{2}$ to $10^{5}$ seconds [2]. Due to their nature BICCs are expected to contribute to all skew and normal multipoles and give rise to a time dependent sinusoidal field pattern with a periodicity equal to $L_{p}[15]$.

Taking into account the local $B_{\perp}$ for each cable, a global electrical loss measurement during a field cycle B1-B2-B1 at different ramp rates results in an average value of $R_{c}$ and $\tau_{i s}$. Fig. 4 shows the integrated total hysteretic and coupling losses during field cycles between 1.9 and $3.8 \mathrm{~T}$ vice versa for both poles. From these measurements an average value $R_{c}=1.2 \mu \Omega$ and $\tau_{i s}=11 \mathrm{~s}$ is calculated. During the heat treatment at $675^{\circ} \mathrm{C}$ the uncoated $\mathrm{Cu}$ surfaces of the strands are probably sintered together, which results in this very low $R_{c}$. The systematic difference between the poles points to a small difference in the average $R_{c}\left(R_{c l}, R_{c 2}\right)$.

Due to the low $R_{c}$, the internal splice configuration and especially the wide inner cable, both BICCs and ISCCs are the expected sources of relatively large field errors during a field sweep. In fig. 5 the absolute field contribution in the straight part of the magnet of the successive multipole terms during a

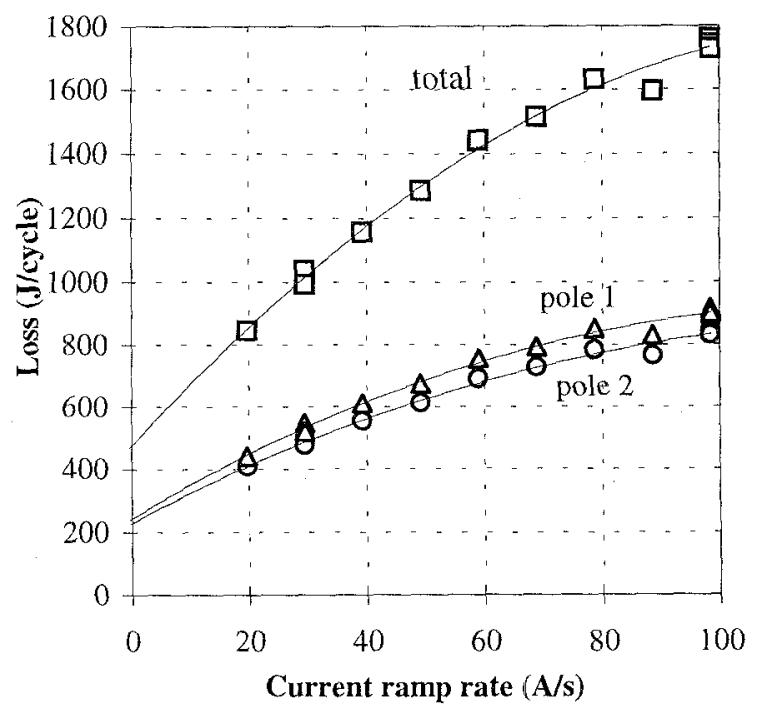

Fig. 4. Measured integrated hysterises and coupling losses as a function of the current ramp rate during a 1.9-3.8-1.9 $\mathrm{T}$ cycle for both poles separately and the total loss. 


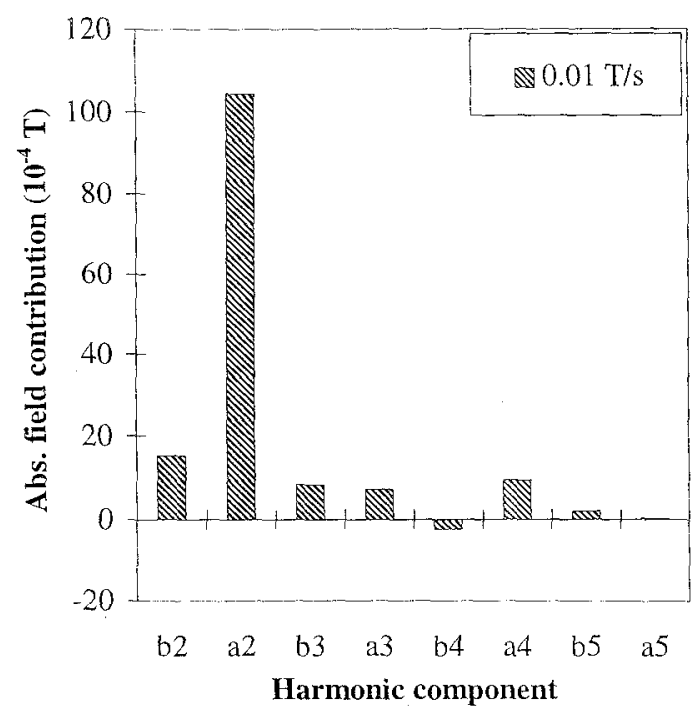

Fig. 5. Absolute field contribution of the higher order multipoles in the straight part during a field sweep from $1.9-5.7 \mathrm{~T}$ with $0.01 \mathrm{~T} / \mathrm{s}$

field sweep from 1.9 to $5.7 \mathrm{~T}$ with $0.01 \mathrm{~T} / \mathrm{s}$ are presented. The skew quadrupole field of $10^{-2} \mathrm{~T}$ is at least 2 orders higher than the tolerable value of $10^{-4} \mathrm{~T}$ and must be attributed to ISCCs proportional to $\mid 1 /\left(R_{c 1}\right)-\left(1 /\left(R_{c 2}\right) \mid\right.$ and to BICCs. This is also reflected by the relatively large $\mathrm{a}_{4}$ contribution. The remaining even and skew odd fields must be attributed to the BICCs. With the known cable parameters and the experimentally value of $R_{c}$ normal odd contributions of $10^{-3} \mathrm{~T}$ for $b_{3}$ and $10^{-4} \mathrm{~T}$ for $b_{5}$ can be expected.

The results of static field measurements are presented in fig. 6. At low field of $0.4 \mathrm{~T}$ the $\mathrm{b}_{3}$ of $5 \cdot 10^{-3}$ is caused by filament magnetisation, due to the $40 \mu \mathrm{m}$ filament diameter and the extremely high $J_{c}$ at low fields. For this conductor saturation of the filaments occurs at 1.2-1.5 T. The large normal and skew quadrupoles might point to a combination of a small misalignment of the coils, asymmetry in the permanent

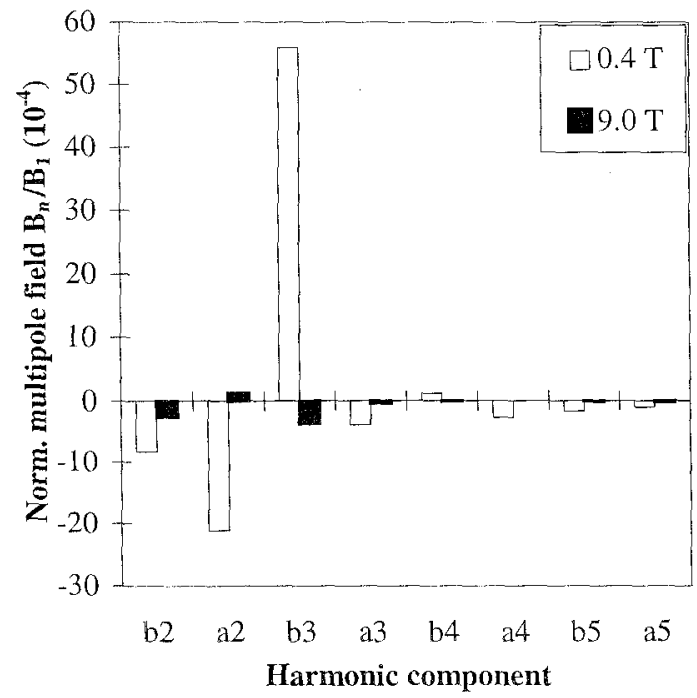

Fig. 6. Normalised multipoles $b_{n}\left(=B_{n} / B_{1}\right)$ at low field $(0.4 \mathrm{~T})$ and high-field (9T) in the straight part. vertical gap between the yoke halves or a non-uniform current distribution due to the soldering connections.

The ramp rate sensitivity of the quench current has been measured in 2 arrangements. In the standard arrangement (labelled WP=without precycle) the current is ramped from zero till the quench. In the second arrangement (labelled $\mathrm{P}=$ precycled) the current is ramped to an intermediate level of 8-11 kA at a moderate speed. After decay of the ISCC's and the BICC's in about 10 minutes the current is ramped down to $2 \mathrm{kA}$ and immediately ramped up to the quench with the desired ramp rate. In this arrangement the BICC's generated in the down ramp are partially compensated in the upramp due to their long relaxation time. As a result the quench current with $\mathrm{P}$ is expected to be higher than with WP, depending on the amplitude of the BICC's. In fig. 7 these results are presented and show a severe reduction of the quench current at higher ramp rates. Especially at low ramp rates between 5 and $50 \mathrm{~A} / \mathrm{s}$ mainly the BICC's are responsible for the reduced quench current. At high ramp rates the temperature rise due to both magnetisation and coupling losses becomes the dominant source of reduction, which would lead both curves to coincide.

At high ramps rates large voltage spikes $(-80 \mathrm{mV})$ across the poles at discrete current levels are observed without quenching the magnet. This confirms the expectation that due to the large BICCs strands become saturated and current redistribution occurs, which in fact illustrates at the same time the high stability margin of the conductors.

\section{Temperature development}

During all field sweep events the temperature development in the inner layer conductor blocks have been recorded. After a second test run at the end of this year more detailed and clarifying results will become available. As an illustration fig. 8 shows the temperatures during a field sweep from the virgin state to the quench at a ramp rate of $0.13 \mathrm{~T} / \mathrm{s}$. Due to the large magnetisation losses a large temperature rise at low

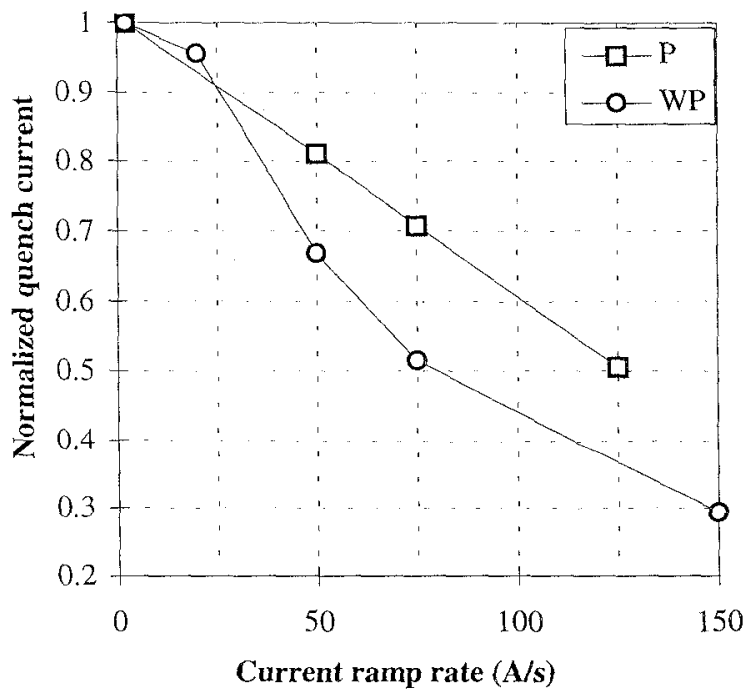

Fig. 7. Normalised quench current $I_{4}(d I / d t) / I_{4}(0)$ as a function of the current ramp rate with (P) and without precycle (WP). 


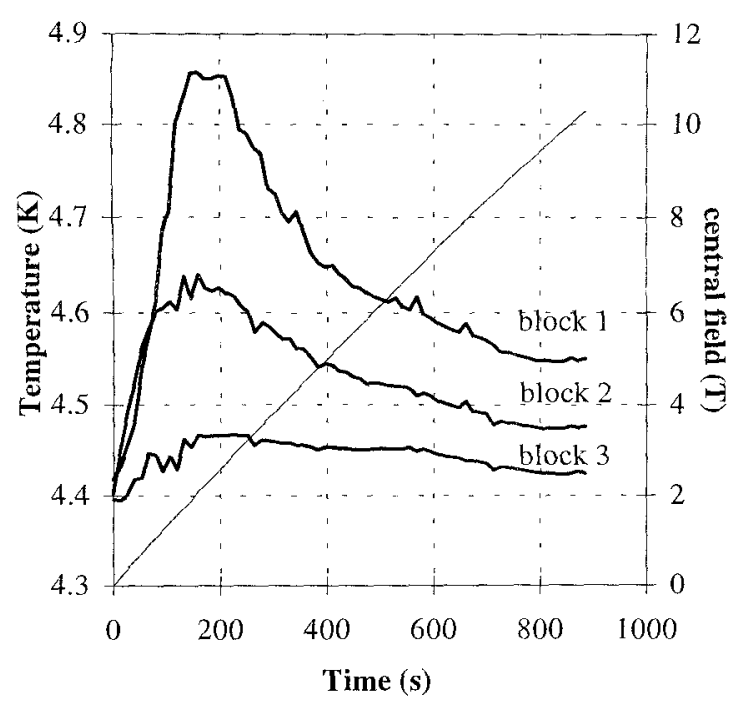

Fig. 8. Measured temperature development in the inner layer blocks (see fig. 2) during a field sweep from $0-10.5 \mathrm{~T}$ at a speed of $0.013 \mathrm{~T} / \mathrm{s}(20 \mathrm{~A} / \mathrm{s})$.

fields occurs. At the end of the ramp the magnetisation contribution is negligible and only the coupling losses determine the local temperatures. Taking into account the modelled loss power, the surface heat transfer to the helium $\left(q=3 \cdot(\Delta T)^{2.5} \mathrm{~W} / \mathrm{cm}^{-2}\right)$ and the heat conductivity of the impregnated glass/mica-glass insulation $\left(\lambda=10 \cdot T \mathrm{mWm}^{-1} \mathrm{~K}^{-1}\right)$ a temperature in block 1 of $4.52 \mathrm{~K}$ is calculated, which agrees very well with these measurements. These unique in-situ experiments show the reliability of the thermometer lay-out and are a valuable tool in the study of the thermal behaviour and stability at cable level.

\section{CONCLUSIONS FROM THE MSUT PROGRAM}

The obtained record field of $11 \mathrm{~T}$ at $4.4 \mathrm{~K}$ at first excitation with the dipole magnet MSUT illustrates the potential of $\mathrm{Nb}_{3} \mathrm{Sn}$ conductors in general and powder-in-tube conductors in particular. It is the first real breakthrough towards fields well above $10 \mathrm{~T}$. This result proves that the developed design and manufacturing concepts provide reliable and good performing coils. Though the manufacturing of a $\mathrm{Nb}_{3} \mathrm{Sn}$ magnet remains a tedious process which demands total commitment, this program has shown that the technology for reliable $\mathrm{Nb}_{3} \mathrm{Sn}$ coil production has evolved to a mature level.

For the highly efficient 2 layer $\cos (\theta)$-design the maximum reachable field strength amounts to about $15 \mathrm{~T}$. This limit is imposed by manufacturing abilities (a further increase of the size of the inner cable impedes production of sound coils) and by the maximum allowable transverse coil stress of about $200 \mathrm{MPa}$.

A high $J_{c}$ of the strand in itself is no guarantee for a good cable performance but greatly improves stability. Severe $I_{c}$ degradation due to filament damage during cabling has been observed in this program. Despite this initial reduction the MSUT cables did not show enhanced stress sensitivity during magnet operation, which confirms the mechanical stability of the PIT-conductor. For each particular $\mathrm{Nb}_{3} \mathrm{Sn}$ application the first development step must be an optimisation of the strand and cable lay-out with respect to the specific operating conditions.

The clear influence of the large magnetisation and coupling currents on the field quality, the losses and the associated temperature development in the coils and finally the ramp rate sensitivity of the quench current are caused respectively by the large filament diameter, the cable dimensions and the low interstrand resistance $R_{c}$. Reduction of the coupling currents by increasing $R_{c}$ is imperative. This can be achieved by insertion of a resistive core between the rows of strands while keeping $R_{a}$ low in order to maintain sufficient thermal and electrical stability. These demands exclude coating of the individual strands with a resistive layer. Further development towards smaller filaments while maintaining a high $J_{c}(B, T)$ is necessary to reduce the magnetisation effects on the field quality.

Just because the MSUT magnet exhibits large ISCCs and BICCs it may serve as a perfect object to study in-situ the characteristic properties of the BICCs, which still cause problems in high-field accelerator magnets.

\section{V. $\mathrm{NB}_{3}$ SN ACCELERATOR MAGNET DEVELOPMENTS}

Presently only one other $\mathrm{Nb}_{3} \mathrm{Sn}$ accelerator dipole model magnet is being realised, the $13 \mathrm{~T}, 4$ layer $\cos (\theta)$ single aperture magnet D20 at LBNL, utilising internal-tin conductors [16]. Characteristic design points different from the MSUT magnet are the small cables (easy to wind, low operating current, higher self-induction), no internal splices, a novel outer shell technique, glass fibre insulation and an optimised coil head lay-out with accurately machined coil head support elements and end pieces. Its completion and first cool-down is expected autumn this year.

To obtain field levels above $15 \mathrm{~T}$ new design concepts have to be developed to overcome the excessive cable dimensions and internal stresses and possibly cooling limitations. Though less efficient and probably more difficult to produce and protect, several twin-bore block designs are proposed that are compliant to these severe demands. They show promising perspectives, provided that the expected problems with respect to structural materials and magnet protection can be solved. An interesting aspect of these designs is the parallel-to-the-wide-side orientation of the field lines, thus relaxing the $R_{c}$ and $R_{a}$ demands [17],[18].

A $300 \mathrm{~T} / \mathrm{m}, 2$ layer $70 \mathrm{~mm}$ bore $\mathrm{Nb}_{3} \mathrm{Sn}$ LHC quadrupole has been designed by the INFN/CERN collaboration, based on an internal-tin conductor [19]. Recently this quaddedicated experimental rectangular Rutherford cable showed a non-Cu $J_{c}$ of $1490 \mathrm{~A} / \mathrm{mm}^{2} @ 11 \mathrm{~T}, 4.2 \mathrm{~K}, 5 \mathrm{MPa}$ and a nonpermanent $I_{c}$. degradation of only $7 \%$ at $200 \mathrm{MPa}$ transverse stress.

Irrespective of the magnet design a further increase in $J_{c}$ is necessary to attain $15 \mathrm{~T}$. The developments for various types of conductors in the last years are very promising, as illustrated by the enhancement of both the $B_{c^{2} 2}$ and $J_{c}>15 \mathrm{~T}$ with the ternary $(\mathrm{NbTa})_{3} \mathrm{Sn}$ conductors.

For the internal-tin and bronze type conductors there still exists a certain trade-off between $J_{c}$ and filament size [20], [21]. The powder-in-tube technology seems to be very promising with respect to this point. Experimental PIT 
$\mathrm{Nb}_{3} \mathrm{Sn}$ strands with $10 \mu \mathrm{m}$ filaments exhibiting a $J_{c}$ of $2200 \mathrm{~A} / \mathrm{mm}^{2} @ 10 \mathrm{~T}, 4.2 \mathrm{~K}$ have already been produced [22].

A much better understanding of cabling degradation with respect to the conductor type, strand and cable lay-out and cabling process parameters is required to profit optimally from the high $J_{c}$ of $\mathrm{Nb}_{3} \mathrm{Sn}$. So far no general guidelines for a specific conductor type nor cable design exist. At present a modest reduction of $5-10 \%$ is found to be acceptable [23].

Also the transverse stress sensitivity of the $I_{c}$ of $\mathrm{Nb}_{3} \mathrm{Sn}$ Rutherford cables still cannot be predicted from general principles. Despite the many experimental results from different kind of cables (size, number of strands, compaction, keystoned or rectangular) using different types of conductor, no convincing correlation between the ruling parameters and the permanent and non-permanent $I_{c}$. reduction has been found yet [24]. Nevertheless a non-permanent $I_{c}$ reduction of $4-6 \%$ at $100 \mathrm{MPa}$ and $7-10 \%$ at $200 \mathrm{MPa}$ is accepted as good, provided that it is accompanied by a negligible permanent reduction. There is no predictable relation between the transverse stress sensitivity of bare wires and fully impregnated cables because of the incomparable stress distribution. Many experiments at the University of Twente test facility have learned, that the most insensitive cabled types seem to be the bronze and the PIT conductors. Internal-tin cables have shown both excellent behaviour as well as large degradation. A detailed and systematic study on this issue is being carried out at in co-operation with LBNL in which an important correlation between the initial degradation after cabling and the stress sensitivity of internal-tin cables has been found [24].

Without any measures the heat treatment will result in a very low interstrand resistance $R_{c}$, resulting in large ISCCs and BICCs and hence large time dependent field errors. Insertion of a resistive metal core between the rows of strands enables independent control of $R_{c}$ and $R_{u}$ [25], [26]. However, core insertion also affects the thermal, mechanical and electrical stability. For NbTi cables this issue is being investigated, but so far no experiences with $\mathrm{Nb}_{3} \mathrm{Sn}$ cables have been reported.

In the coming three years the existing MSUT collaboration will continue to develop and build an $88 \mathrm{~mm}$ wide bore $10 \mathrm{~T}$ $\mathrm{Nb}_{3} \mathrm{Sn}$ model LHC separation magnet, according to the design and manufacturing concepts developed for the MSUT magnet. To arrive at the required field quality, both reduction of the filament size while maintaining a high $J_{c}$ and control of $R_{c}$ and $R_{a}$ will be pursued in this program. When this nextstep R\&D project is successful it becomes feasible to incorporate a few $\mathrm{Nb}_{3} \mathrm{Sn}$ separator dipole magnets in the $\mathrm{LHC}$ insertion region.

\section{ACKNOWLEDGMENT}

The authors would like to thank gratefully R. Perin, D. Leroy, P. Sievers, L. Walckiers, A. Siemko, L. Bottura A. Verweij and all members of the model-magnet test facility at CERN for their contribution to the MSUT program.

\section{REFERENCES}

[1] A. Siemko et al.,"Power test results of the first LHC second generation superconducting single aperture $1 \mathrm{~m}$ long dipole magnets", presented at the 16th ICEC/ICMC conference, 1996, Kitakyushu, Japan

[2] A.P. Verweij, "Electrodynamics of superconducting cables in accelerator magnets", PhD. Thesis, University of Twente, 1995.

[3] M. Wilson et al.," Rutherford cables with anisotropic transverse resistance", presented at this conference.

[4] ] The PIT $\mathrm{Nb}_{3} \mathrm{Sn}$ conductor has been developed and produced by ECN, the Netherlands. The production and further development has been taken over by Shape Metal Innovation, Enschede, the Netherlands.

[5] H.H.J. ten Kate et al., "Critical current measurements of prototype cables for the LHC up to $50 \mathrm{kA}$ and between 7 and $13 \mathrm{~T}$ using a superconductor transformer circuit", Proceedings of MT11, Tsukuba, 1992, p. 60 .

[6] H.H.J. ten Kate et al., "Development of a 'twin aperture' $10 \mathrm{~T} \mathrm{Nb}_{3} \mathrm{Sn}$ dipole magnet for the CERN LHC', Proceedings of MT1I, Tsukuba, 1992 , p. 66.

[7] A. den Ouden et al., "An experimental $11.5 \mathrm{~T} \mathrm{Nb} 3 \mathrm{Sn}$ LHC type of dipole magnet", IEEE Trans. Mag. vol. 30, 1994, p. 2320

[8] A. den Ouden, W. van Emden and H.H.J. ten Kate, "Analysis of the mechanical behaviour of an $11.5 \mathrm{~T}$ LHC dipole magnet according to the ring collar concept", IEEE Trans. Mag. vol. 28, 1992, p. 331

[9] A. den Ouden and H.H.J. ten Kate, "Thermal conductivity of mica/glass insulation for impregnated $\mathrm{Nb}_{3} \mathrm{Sn}$ windings in accelerator magnets", Cryogenics vol. 34, p 385.

[10]L. Walckiers, "The harmonic-coil method", CERN accelerator school on magnetic measurement and allignment, 1992, p. 138.

[11]J. Billan, A. Siemko, L. Walckiers, R. Wolf, "Quench localisation in the superconducting model magnets of the LHC by means of pick-up coils", IEEE Trans. on Applied Superconductivity vol. 5, 1995, p. 1028

[12]F. Gömöry and L. Cesnak, "Loss and magnetisation measurement of superconducting magnets pulsed at very low fields", Cryogenics vol. 25,1985 , p. 375

[13]H.W.Weijers, H.H.J. ten Kate, J.M. van Oort, "Critical current degradation in $\mathrm{Nb}_{3} \mathrm{Sn}$ cables under transverse pressure", IEEE Trans. on Applied Superconductivity vol. 3, 1993. p. 1334.

[14]A. Asner, R. Perin, S. Wenger, F. Zerobin, "First Nb3 3 Sn 1 meter long superconducting dipole model magnet for LHC breaks the $9.5 \mathrm{~T}$ threshold", Proceedings of MTI1, Tsukuba, 1992, p. 36

[15] L. Bottura and L. Walckiers, 'Evidence of Boundary Induced Coupling Currents in LHC models", presented at this conference.

[16]D. Dell' Orco et al., "Design of the $\mathrm{Nb}_{3} \mathrm{Sn}$ dipole $\mathrm{D} 20$ ", IEEE Trans. on Applied Superconductivity vol. 3, 1993, p. 82

[17]P. McIntyre et al., "Design of a $16 \mathrm{~T}$ dual dipole", presented at this conference.

[18]J.M. van Oort and R.M. Scanlan, "Design of a $16 \mathrm{~T} \mathrm{Nb}_{3} \mathrm{Sn}$ twin bore accelerator dipole with a window-frame conductor lay-out", IEEE Trans. on Applied Superconductivity vol. 5, 1995, p. 1008.

[19]L. Rossi, "INFN work on quads", collected papers of the 32 nd workshop of the Eloisatron Project, Erice, 1996.

[20]M. Thöner, "New conductor development at VAC", collected papers of the 32 nd workshop of the Eloisatron Project, Erice, 1996.

[21] L.R. Motowidlo, "Superconducting materials activities at IGC", collected papers of the 32 nd workshop of the Eloisatron Project, Erice, 1996

[22]E.M. Hornsveld et al., "Development of ECN-type $\mathrm{Nb}_{3} \mathrm{Sn}$ wire towards smaller filament size", Advances in Cryogenic Engineering, vol. 34, 1987, p. 493.

[23]R. Scalan, "Mechanical properties of $\mathrm{Nb}_{3} \mathrm{Sn}$ strands and cables", collected papers of the 32nd workshop of the Elois. Project, Erice, 1996.

[24] J.M. van Oort, Thesis, University of Twente, to be published.

[25]E.W. Collings et al., "Magnetic studies of AC losses in pressurized Rutherford cables with coated strands and resistive cores", $A d v$. in Cryogenic Engineering vol. 42, 1996, in press

[26]E.W. Collings et al., "Suppression of eddy current loss in bare-copper Rutherford cables using stainless steel cores of various thickness", presented ath the 16 th ICEC/ICMC conference, 1996, Kitakyushu, Japan 